\title{
Geo-cosmic Universal X-structure of Solar System Planets and Satellites in Applying to Exoplanet Systems
}

\author{
A. G. Syromyatnikov \\ Department of Physics, St. Petersburg University, St. Petersburg, Russia
}

Email address:

alsyromyatnikov@mail.ru

\section{To cite this article:}

A.G. Syromyatnikov. Geo-cosmic Universal X-structure of Solar System Planets and Satellites in Applying to Exoplanet Systems. International Journal of Systems Science and Applied Mathematics. Vol. 2, No. 1, 2017, pp. 10-24. doi: 10.11648/j.ijssam.20170201.12

Received: October 13, 2016; Accepted: December 22, 2016; Published: January 18, 2017

\begin{abstract}
Given a new unique method of calculating satellite systems approved at all, without exception, 185 satellites of the Solar system. The base of the Geo-cosmic Universal X-structure of Solar system planets and satellites represents a discretecontinuous distribution of satellite and longest rings in axes the density/orbital radius (semi-major axis of the orbit), converted to the scale of the central body. Previously, it was found that in all cases, the maximum radius of the rings is the same as the triple radius of the central body for the border zones of destructive tidal actions with $12 \%$ spread. For Pluto's radius $1184 \mathrm{~km}$ radius of rings on X-structure may range from 4000 to $7000 \mathrm{~km}$. It was far enough away from $12000 \mathrm{~km}$ radius of passage of spacecraft "New horizons". The success of the application of the method of the X-structure is shown here. For some exoplanets within 50 light-years $(15 \mathrm{pc})$ of the Earth by a method of the Geo-cosmic universal X-structure it is studied questions of the definition of the exoplanets parameters: density-diameter etc. Given also the Universal Geo-cosmic X-structure theoretical Levels of the similarity of natural satellites of the Solar system planets. Comparative characteristics between the method of the $\mathrm{X}$-structure on the one hand and the modification of the Lunar systems Titius-Bode law on the other hand given in Appendix A. From the theory, in particularly, follows the mystery similarity between the Mercury and the Earth's Moon. Study of this layer of the undoubted new reality held in Appendix B. On pictures of the surface of Pluto and Charon the crust thickness was determined. Crust of Pluto from pole $21 \mathrm{~km}$. The ice crust Charon thickness at pole is $18 \mathrm{~km}$.
\end{abstract}

Keywords: Exoplanet, Solar System Planets and Natural Satellites, Discrete-Continuous Density Distribution, Pluto Crust Thickness

\section{Introduction}

There is a Galactic Winter on the Earth at present [1]. It's pretty quiet time when nobody no major geological events does not occur except in the local area as it was 10,000 years ago with the isthmus connecting Asia with America (Bering Strait), in the formation of the Straits separating New Zealand, off the East coast of North America, etc. But sometimes comets fall into Jupiter. The observed plasma balls emissions from the area Center of the Galaxy with V Hydrae can coincide with achieving the next Sergey Neruchev's uranium point on solar Galactic orbit [2] that is not expected soon, so you can expect another 20,000,000 years such a life. The attention of researchers of an outer cosmic has attracted discovering exoplanets outside the Solar system [3-6]. For centuries philosophers and scientists supposed that extrasolar planets existed, but there was no way of detecting them or of knowing their frequency or how similar they might be to the planets of the Solar system. Various detection claims made in the nineteenth century were rejected by astronomers. The first confirmed detection came in 1992, with the discovery of several terrestrial mass planets orbiting the pulsar PSR B1257+12 [7]. The first confirmation of an exoplanet orbiting a main-sequence star was made in 1995, when a giant planet was found in a four-day orbit around the nearby star 51 Pegasus. Some exoplanets have been imaged directly by telescopes, but the vast majority has been detected through indirect methods such as the transit method and the radial-velocity method. The first published discovery to receive subsequent confirmation was made in 1988 by the Canadian astronomers Bruce Campbell, G. A. H. Walker and Stephenson Yang of the university of Victoria and the university of British Columbia (Web-site: https://en.wikipedia.org/wiki/Exoplanet). Although they were cautious about claiming a planetary detection, their radial- 
velocity observation suggested that a planet orbits the star Gamma Cepheid. Partly because the observations were at the very limits of instrumental capabilities at the time, astronomers remained skeptical for several years about this and other similar observations. It was thought some of the apparent planets might instead have been brown dwarfs, objects intermediate in mass between planets and stars. In 1990 additional observations were published that supported the existence of the planet orbiting Gamma Cepheid but subsequent work in 1992 again raised serious doubts. Finally, in 2003, improved techniques allowed the planet's existence to be confirmed [7].

Initially, most known exoplanets were massive planets that orbited very close to their parent stars. Astronomers were surprised by these "hot Jupiter's", because theories of planetary formation had indicated that giant planets should only form at large distances from stars. But eventually more planets of other sorts were found, and it is now clear that hot Jupiter's area minority of exoplanets. In 1999, Upsilon Andromeda became the first main-sequence star known to have multiple planets. Others were found subsequently. As of 28 March 2015, a total of 1906 confirmed exoplanets are listed in the Extrasolar Planets Encyclopedia, including a few that were confirmations of controversial claims from the late 1980s. That count includes 1202 (more 2000 at present planetary systems, of which 480 are multiple planetary systems). Kepler-16 contains the first discovered planet that orbits around a binary main-sequence star system.

There are exoplanets that are much closer to their parent star than any planet in the Solar System is to the Sun, and there are also exoplanets that are much further from their star. Mercury, the closest planet to the Sun at 0.4 AU, takes 88 days for an orbit, but the smallest known orbits of exoplanets have orbital periods of only a few hours, e.g. Kepler-70b. The Kepler-11 system has five of its planets in smaller orbits than Mercury's. Neptune is 30 AU from the Sun and takes 165 years to orbit it, but there are exoplanets that are thousands of AU from their star and take tens of thousands of years to orbit, e.g. GU Piscium b.

The orbit of a planet is not centered on the star but on their common center of mass. For circular orbits, the semi-major axis is the distance between the planet and the center of mass of the system. For elliptical orbits, the planet-star distance varies over the course of the orbit, in which case the semimajor axis is the average of the largest and smallest distances between the planet and the center of mass of the system. If the sizes of the star and planet are relatively small compared to the size of the orbit and the orbit is nearly circular and the center of mass is not too far from the star's center, such as in the Earth-Sun system, then the distance from any point on the star to any point on the planet is approximately the same as the semi-major axis.

The radial-velocity and transit methods are most sensitive to planets with small orbits. The Kepler spacecraft has found planets with even shorter orbits of only a few hours, which places them within the star's upper atmosphere or corona, and these planets are Earth-sized or smaller. If a planet is detectable by both the radial-velocity and the transit methods, then both its true mass and its radius can be found. The planet's density can then be calculated. Planets with low density are inferred to be composed mainly of hydrogen and helium, whereas planets of intermediate density are inferred to have water as a major constituent. A planet of high density is inferred to be rocky, like Earth and the other terrestrial planets of the Solar System.

\section{Aims \& Methods}

There are not all exoplanets parameters such as density and radius defines. In all these indeterminate cases it may be possible productive use of the Geo-cosmic universal Xstructure of the Solar system [1]. The X-structure constructed on basis of the theory (see also ref. No 7 at [9]) [1, 8, 10-11] as it tested in [11]. The binding structure is made in a standard way by setting a scale factor $\mathrm{K}$ equal to the relation of the diameter of the central star to the diameter of the Sun. It has been tested on the diameter of the rings of planetsGiants are Saturn, Jupiter and others: in all cases, the maximum diameter of the rings matches the 3 diameters of the planet in accordance with the known value of the boundaries of the zone of tidal destruction. In this way, managed to set the density of Saturn's rings. Within the small standard deviation of $23 \mathrm{mG} \cdot \mathrm{cm}^{-3}$ it is the same as the density of ordinary ice, with minor additives in agreement with observed data measuring the rings coefficient of reflection. There are not any forms of a heavy ice [11].

Originally, Universal Geo-cosmic X-structure of the planets and satellites of the solar system was established to study the processes of differentiation of substances in the solar gravitational field, in the context of the new for me the idea of the integrity of the solar system, on the basis of a new physical value gravitational mobility for atomic dust, imposed by the author in 1998 year in relation to the dense solar plasma. It turned out that the atoms in the Sun gravitation field distributed by law of Archimedes: heavier species are placed closer to the Sun and more light to the periphery. The accumulation of light elements really takes place in the field of distant orbits of the gas giants. Earth with an iron nucleus is located much closer to the Central luminary. On Mercury recently discovered the strongest gravitational anomalies near the equator, which means the accumulation of heavy elements. Along the way I have come to the conclusion (2000-2001) of the possible accumulation of water by oxygen in orbit of Jupiter. This conclusion shortly received confirmation as a result of the discovery of the crust of water ice on Europe, Jupiter's moon. These findings are applicable to other planetary systems, since the Sun is a main sequence star. Consequently, the gravitational differentiation of substances in the gravitational field of other stars should look like, including the characteristics of density.

Lately, as mentioned above, received a large amount of data on the very remote outside solar planetary systems. Mostly they include individual gas giants Jupiter's scale. But there is also a confirmed extrasolar planet Earth scale. Many 
from them too close to the cosmic object that conditions were suitable for life. Our solar system is unique in its kind. Indeed, on the basis of spectral classes, sample bolometric luminosities, masses and radii of stars-dwarfs was calculated (Ksanfomality 1997) at what distance from the star to be placed the planet with spherical albedo, the albedo equal to the Earth's albedo to illumination was equal to known for Earth solar constant. In other words, in such a hypothetical planet would be "normal" terrestrial conditions. It turned out that in all cases, the planet must be at distances within the semi-major axis orbit of Mercury (0,39AU) [12]. Some ideas about the formation of planetary systems from such dust clouds was obtained from the model experiments on computer [12]; It turned out that the Division of the planets' earthly Group (see [3-4]) and the planets-Giants typically: "In all cases at a distance close to 1 AU present planet (one or more) with the weight close to the mass of the Earth». But factors such as excessive speed of collisions are known to make problematic the existence of such planets. Consideration of these long time processes for conditions of the Protoplanetary cloud based on Newton's gravitation equations and the equation of continuity taking into account the diffusion required the introduction of a new concept of gravitational mobility (1998). The magnitude of the gravitational mobility [1] is defined via the electric mobility of ions by replacing the electrical charge of the ion on its mass. It is very small: $4 \cdot 10^{-15}$ seconds for a proton in water. On the other hand in the long-running processes of planetary formation from Protoplanetary cloud over $4500 \mathrm{Ma}$ (million years) chemical elements are able to cross the distance of Mercury from the Sun 0.4 AU in its gravitational field. This shows that the dense part of the solar system from Mercury to Earth covered molecular migration in the gravitational field of a central luminary. In the gravitational field of the giant planets Jupiter, Saturn, Uranus and Neptune the corresponding distance gravity diffusion transfer has order the size of their rings. It gives a visual representation of gravity diffusion transfer now, as the planets rings composed of ice, in which the gravitational mobility well defined in accordance with the above value. Previously for this system of equations of gravity diffusion transfer in the gravitation field I got the exact, asymptotically stable solution of soliton type-the gravity diffusion soliton (GDS) - the width of the front which determines the position of the maximum density of chemical elements in each class in orbit. This value of the width of the front of the gravity diffusion soliton is constant and is determined by the ratio of the diffusion coefficient to the gravitational mobility (and to jump of gravitation field) for which it should be performed the ratio of Einstein as for mobility in an electric field. This leads to the value which is equal to relation of temperature to the mass of the nucleus. It is this dependence of the width of the front of GDS from the mass of the nucleus allows you to present dependence of quantum distribution of Quark-lepton X-structure [1, 8, 1011] as quantum probability density, more precisely, the quantum Green's function) from the mass of nuclei in the form depending of mass density on the size of the semi- major axis of the orbit is still related jump (density). This should take into account the common property of solitons preserve the original envelope, which in this case is the nature of the reporting of the quantum distribution (this is a Green function defined in terms of high-energy processes [9]) for atomic dust of a Protoplanetary cloud. Moreover, the fall of meteorites and asteroids, etc. for a long time makes no significant changes since falling asteroid breaks into atomic dust (little is known on the Earth of ancient meteorite craters).

Basics of Quark-lepton X-structure can be found in [8]. Difficult tasks I usually decide two and sometimes in three ways. Here, I must limit myself to only one, namely, the approach based on an accurate salvation non-linear Schrödinger equation with some potential (1990) with asymptotic $U \sim|\Psi|^{2}$ and $U \sim|\Psi|$. In the first case, the solution is approximated by solution famous Schrödinger equation with cubic nonlinearity and in the second case, the decision of the Schrödinger equation with quadratic nonlinearities. The second occasion reduced system of linear Schrodinger equations for electron and nucleus in the collective electric field that meets the Poisson's equation with the source type of the sum of the electron and ion charge densities in conditions of partial neutralization. There are some known applications of the non-linear Schrödinger equations to nuclear physics (see, for example ${ }^{1}$ ). It is essential for applications that both [8], and in the above case, the nonlinear Schrödinger equation the system is formed with ten peaks describe the distribution of stable decay products heavy nuclei together with the distribution of their instant gamma radiation production after interaction with one thermal neutron. Also in both cases, a separate peak is not Gaussian type and, in the case of [8] imagine, in the form of dual and triple quasi Gaussian peaks, which are also not possible to divide, as it is impossible to see quarks. In applying for the X-structure of the planets and satellites of the solar system, as well as in geology, in fact the original Quark-lepton X-structure turned out to behalf fragmented: cut-off elements everywhere going on weight 150 AUM, above which theoretically should be only $\alpha$ decay elements. This can be seen as a confirmation of the theory of $\alpha$-decay. Fragmented medium and heavy chemical elements in the complex are captured more light on the non linear soliton mass doubling mechanism: for example, heavy item weighing 225 captured Cd-112, etc.

The development approach [1, 10-11] when you identify the provisions of satellites with the jumps points Geo-space $\mathrm{X}$-structure, the relevant provisions of the differential distribution density jumps, it was considered and correspondence of the density of satellites to theoretical values. When this, if the density of the satellite is within the density variations on jump, the deviation from the theory can be considered zero. A nonzero density variance will be counted from the boundary values of the density on the jump, when the density of the satellite goes beyond the boundaries of settlement density variations on the jump.

Almost simultaneously with the formulation of the

${ }^{1}$ Brenner, M., Gridnev, K.A., Belov, S.E. 2002, Phys. Atomic Nuclei, 65, 612. 
foundations of an Universal Geo-space X-structure of the solar system, by virtue of universality solitons found in all aggregation states of substances, including plasma, was initiated the Universal Geo-space X-structure of the Earth crust and upper mantle [1]. There are many geology terms in the X-structure of the solar system. In Appendix B shows the thickness of the crust of Pluto and Charon, received as result of processing the method X-structure pictures of its surface, made by the New Horizons spacecraft.

\section{Results}

By the beginning of 2015, astronomers have identified a total of 65 exoplanets within 50 light-years $(15 \mathrm{pc})$ of the Earth, but the existence of at least another 35 unconfirmed exoplanets has been proposed. This corresponds to only 35 stars with confirmed planetary systems (and eleven with only unconfirmed exoplanets) of the around 1,400 stars that are estimated to be located within 50 light-years. Reports of planetary systems first came in 1996 for three stars located over 40 light-years away: 55 Chancre, Upsilon Andromeda and 47 Ursine Majors. Since 1999, more planets have been reported, including a total of five planets revolving around 55 Cancri, and four planets around Gliese 876 and Upsilon Andromeda. Eight star systems have three confirmed planetary partners, four others have only two planets, while the remaining 20 systems have only one confirmed planet. A total of seven planets has been suggested for Gliese $667 \mathrm{C}$ (only two have been confirmed), six around HD 40307 (three confirmed).

Gliese $581 \mathrm{c}$ or G1 $581 \mathrm{c}$ is a planet orbiting the red dwarf Gliese 581. It is the second planet discovered in the system and the third in order from the star. With a mass at least 5.5 times that of the Earth it is classified as a super-Earth (a planet of 1 to 10 Earth masses). At the time of discovery, it was the smallest known extrasolar planet around a mainsequence star but on April 21, 2009, another planet orbiting Gliese 581, Gliese 581 e, with an approximate mass of 1.9 Earth masses, was announced. Gliese $581 \mathrm{c}$ gained interest from astronomers because it was reported to be the first potentially Earth-like planet in the habitable zone of its star, with a temperature right for liquid water on its surface, and by extension, potentially capable of supporting extremophile forms of Earth-like life. However, further research casts doubt upon the planet's habitability. It is tidally locked (always faces the parent star with the same face) so if life had a chance to emerge, the best hope of survival would be "the twilight zone". In astronomical terms, the Gliese 581 system is relatively close to Earth, at 20.3 lighty ears (192 trillion km or 119 trillion miles) in the direction of the constellation of Libra. The star Gliese 581 (see the list of open exoplanets for 2010 year) belongs to the spectral class G2V, more cold from 2000 to $3500^{\circ} \mathrm{C}$, as the Sun is $5778 \mathrm{~K}$. Assuming that its mass and size of the Sun, you can calculate the density of the extrasolar planet Gliese $581 \mathrm{c}$, based on the universal Geocosmic X-structure of Solar system [1, 10-11] on Figure 1.

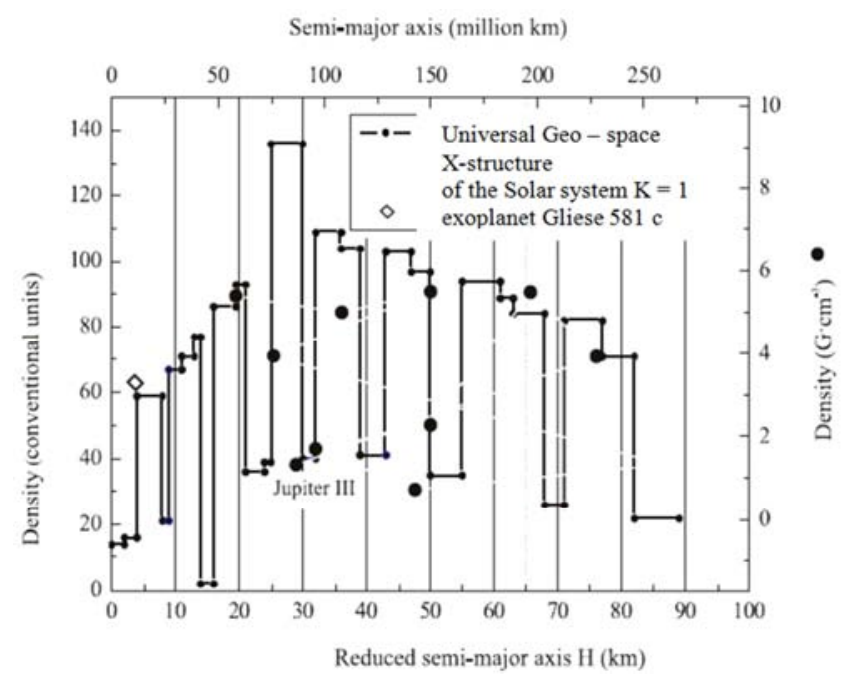

Figure 1. Gliese 581c (diamond, left).

The extrasolar planet Gliese $581 \mathrm{c}$ of the orbits radius 10.5 million kms hould defend from its central luminaries to 3.15 $\mathrm{km}$ the reduced length. According to the Figure 1 of the universal Geo-cosmic X-structure of the Solar system graph in axes density/semi-major axis of the orbit the Gliese $581 \mathrm{c}$ position is precisely on the density exoplanets $3.6 \mathrm{G}^{\cdot} \mathrm{cm}^{-3}$ as icy or watery planet-ocean for its radius of about 2 Earth radii, whereas if it is a rocky planet with a large metal kernel, the density is much higher and its position in Figure 1 is not consistent.

On these established here features an extrasolar planet similar to Europe-the Moon of Jupiter, only larger. Jupiter III indicate the position of Jupiter in the third Layers of Figure 2.

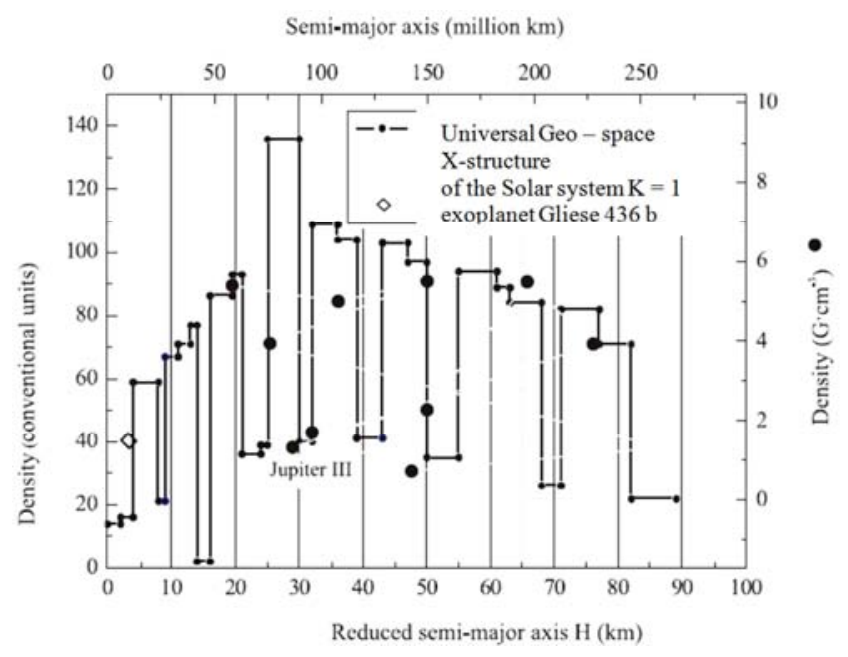

Figure 2. Exoplanet Gliese 436 b (diamond, left).

The exact values of the density of the extrasolar planets are defined when you know their mass and radius. Only three exoplanets radii were measured by the transit method: Gliese 436 b-0.365 RJ, 55 Cancrie-0.178 RJ and GJ 1214 b-0.238 RJ. In Figure 2-4 these exoplanets are plotted on a graph of the universal Geocosmic X-structure of the Solar system [1]. 


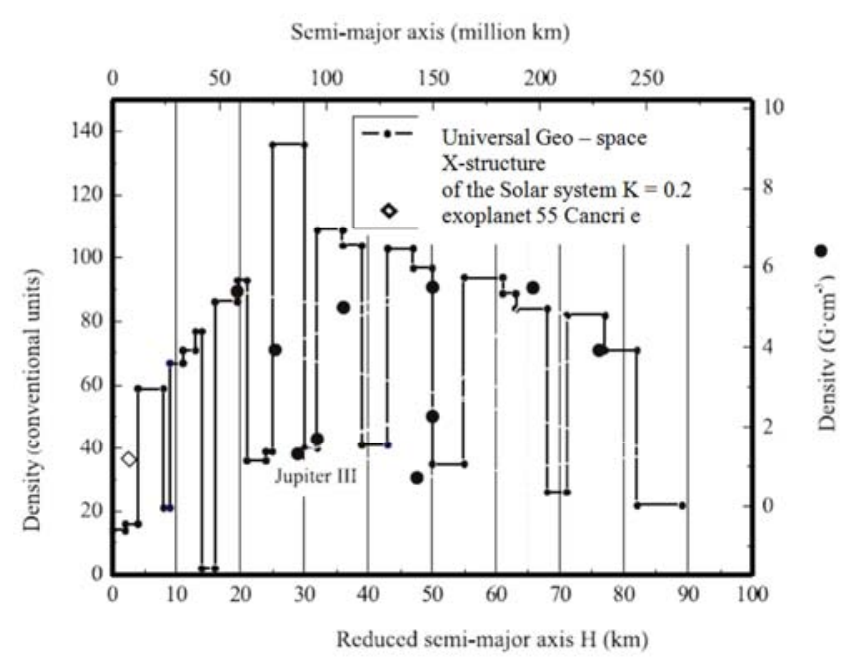

Figure 3. Exoplanet 55 Cancri e (diamond, left).

This exoplanet is far from the central star radius at 0.42 the radius of the Sun on $3.5 \mathrm{~km}$ the reduced semi-major axis of the orbit, expressed with the scale coefficient $\mathrm{K}=0.42$ reduction proportional to the radius of the star relative to the radius of the Sun. This factor to bind the X-structure scale is determined by the fact that the known limit of the zone of destructive deformation is proportional to the gravitational radius of central body. On the Figure 2 exoplanets point is exactly on the Geo-cosmic X-structure graph. Jupiter III indicate the position of Jupiter in the third Layers of Figure 2.

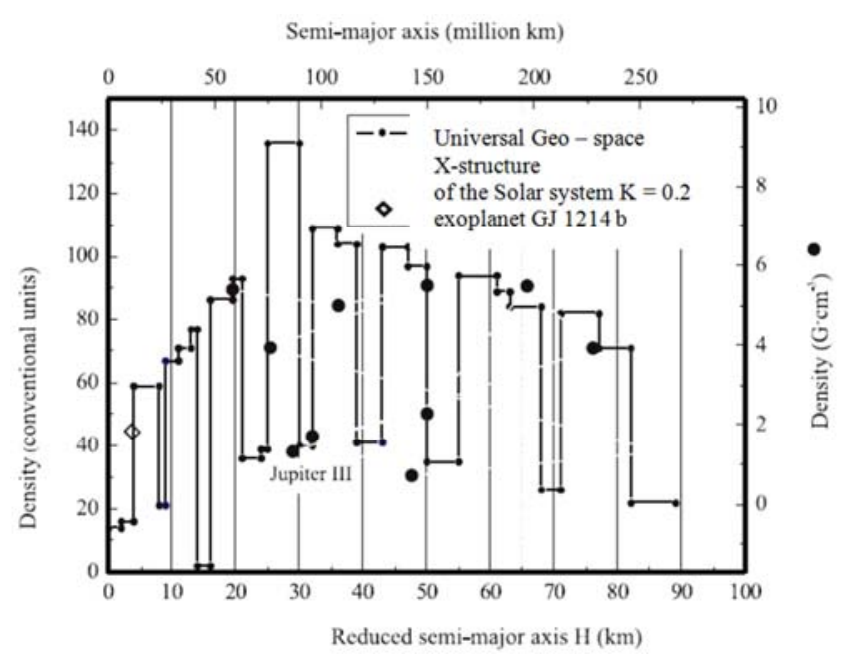

Figure 4. GJ 1214 b (diamond, left).

This planet is from the central star radius 1.15 of the Sun's radius to $1 \mathrm{~km}$ of the reduced semi-major axis of the orbit, without recalculation of the rate of reduction as to the order of 1 in proportion to the radius of the star relative to the radius of the Sun. On the Figure 3 the exoplanet's point position is satisfactorily on the Geo-cosmic X-structure graph within the permissible deviation of the length.

And, finally, -the third planet GJ 1214 b, extends from the Earth to 42 light years. This planet is far from the central star radius 0.2 of the Sun's radius on $3.6 \mathrm{~km}$ the reduced semimajor axis of the orbit in terms of reduction coefficient $\mathrm{K}=$
0.2 is proportional to the radius of the star relative to the radius of the Sun. On the Figure 4 exoplanet's point is exactly on graph of the Geo-cosmic X-structure within the permissible deviation of the length despite of a fairly high density. As can be seen in all three cases, parametric exoplanets points on scheme well consistent with the universal Geo-cosmic structure [10-11] for various radii of the central star.



Figure 5. 82 G. Eridani b, c, d (3 diamonds, left).

The 82 Eridani star system weight 0.70 the Sun's mass and radius of the 0.92 Sun's radius distant 19.7 light years from Earth discovered and supposed three exoplanets $82 \mathrm{G}$. Eridani b, c, d. From the measured albedo 0.3, the surface temperature of exoplanets $115^{\circ} \mathrm{C}$ is allowed this stellar system top grade of habitability. The major semiaxis of orbits in a way binding (scaling factor $\mathrm{K}=0.92$ order 1 ) identified as $6.58 \pm 0.1,11.1 \pm 0.16,19.0 \pm 0.32 \mathrm{~km}$ for extrasolar planets $\mathrm{b}$, c, d respectively. Real these values can be defined on the upper scale Figure 5, which shows that all parametric points of extrasolar planets extremely well on the theoretical curve $\mathrm{X}$ - structure with all the uncertainty values of density, because large half-orbit exactly coincided with the features (jumps) of density of the X-structure. The range of density values of extrasolar planets $b, c, d$ respectively their masses [https://en.wikipedia.org/wiki/Exoplanet] $(2.52 \pm 0.3) \mathrm{M}_{\text {Earth }}$, (2.52 \pm 0.3$) \quad \mathrm{M}_{\text {Earth }}$ and (4.7 \pm 0.6$) \quad \mathrm{M}_{\text {Earth }}$ are exactly corresponded to the weight jumps diapasons of the $\mathrm{X}$ structure. In this field within the spread the Universal Geocosmic X-structure of the planets in the Solar system gives the following values for the radii of extrasolar planets b, c, d 82 Eridani-10880km, $9860 \mathrm{~km}$ and $10720 \mathrm{~km}$ respectively.

In the latter two cases, the theory gives the valid interval radii values exoplanets from $9470 \mathrm{~km}$ up to $10250 \mathrm{~km}$ and from $10270 \mathrm{~km}$ to $1170 \mathrm{~km}$. Important when comparing theory with observed three exoplanets orbit parameters 82 Eridani is the largest major axes all three exoplanets are exactly at the boundary set theory. The density closest to the 82 Eridani extrasolar planet is additional fixed by the largest plateau of the first peak Figure 2 due to the small off set to the left. However, the theory is valid and the interval of less 
than $3 G^{\cdot} \mathrm{cm}^{-3}$, while the more distant orbits, one of which coincided with the orbit of Mercury, the theoretically permission interval of density is small and coincided with the experimental dispersion. Because the values of the density less than $1 G^{\cdot} \mathrm{cm}^{-3}$ an extrasolar planet is a gas balloon (not discussed), theoretical limits on the density of the confirmed exoplanets 82 Eridani b would be:

$$
\text { 1.4 } \mathrm{R}_{\text {Earth }}<\mathrm{R}_{\text {exo }}<2.2 \mathrm{R}_{\text {Earth }}
$$

If the density is more than $6 \mathrm{G} \cdot \mathrm{cm}^{-3}$, it can be said with certainty about the existence of heavy chemical elements.

The nearest planet found recently in the system of red dwarf Proxima Centauri b [13] with a radius of $1 / 7$ of the Sun's radius. Orbital radius exoplanets is equal to $1 / 20 \mathrm{AU}$ is 5 times the diameter of the Sun. X-structure of this exoplanet Proxima Centauri b has great similarities with Mercury, even at $5 \mathrm{G} / \mathrm{cm}^{3}$ density.

\section{Summary}

(1) Assuming that Gliese's mass and size of the Sun, you can calculate the density of the extrasolar planet Gliese $581 \mathrm{c}$, based on the universal Geo-cosmic X-structure of Solar system. The Gliese $581 \mathrm{c}$ position is precisely on the exoplanets density $3.6 \mathrm{G}^{\cdot} \mathrm{cm}^{-3}$ as icy or watery planet-ocean for its radius of about 2 Earth radii, where as if it is a rocky planet with a large metal kernel, the density is much higher and its position in Figure 1 is not consistent.

(2) The exact values of the density of the extrasolar planets are defined when you know their mass and radius. Only three exoplanets radii were measured by the transit method: Gliese 436 b-0.365 RJ, 55 Cancrie- 0.178 RJ and GJ 1214 b- 0.238 RJ. In Figure 2-4 these exoplanets are plotted on a graph of the universal Geocosmic X-structure of the Solar system. On the Figure 2 exoplanets point is exactly on the Geo-cosmic $\mathrm{X}$-structure graph.

(3) On the Figure 3 the exoplanet's point position is satisfactorily on the Geo-cosmic X-structure graph within the permissible deviation of the length.

(4) On the Figure 4 exoplanet's point is exactly on graph of the Geo-cosmic X-structure within the permissible deviation of the length despite of a fairly high density. As can be seen in all three cases, parametric exoplanets points on scheme well consistent with the universal Geo-cosmic structure [10-11] for various radii of the central star.

(5) The 82 Eridani star system weight 0.70 the Sun's mass and radius 0.92 of the Sun's radius distant 19.7 light years from Earth discovered and supposed three exoplanets $82 \mathrm{G}$. Eridani b, c, d. From the measured albedo 0.3, the surface temperature of exoplanets $115^{\circ} \mathrm{C}$ is allowed this stellar system top grade of habitability. The major semi axis of orbits in a way binding on the radius of the central star (scaling factor $\mathrm{K}$ masses $(2.52 \pm 0.3) \mathrm{M}_{\text {Earth }},(2.52 \pm 0.3) \mathrm{M}_{\text {Earth }} \mathrm{a}$ $\mathrm{nd}(4.7 \pm 0.6) \mathrm{M}_{\text {Earth }}$ are exactly corresponded to the weight jumps diapasons of the $\mathrm{X}$-structure. In this field within the spread the Universal Geo-cosmic X-structure of the planets in the Solar system gives the following values for the radii of extrasolar planets b, c, d 82 Eridani- $10880 \mathrm{~km}, 9860 \mathrm{~km}$ and $10720 \mathrm{~km}$ respectively.

(6) Important when comparing theory with observed three exoplanets orbit parameters 82 Eridani is the largest major axes all three exoplanets are exactly at the boundary set theory. The density closest to the 82 Eridani extrasolar planet is additional fixed by the largest plateau of the first peak Figure 2 due to the small off set to the left. However, the theory is valid and the interval of less than $3 \mathrm{G}^{\cdot} \mathrm{cm}^{-3}$, while the more distant orbits, one of which coincided with the orbit of Mercury, the theoretically permission interval of density is small and coincided with the experimental dispersion. Because the values of the density less than $1 G^{\cdot} \mathrm{cm}^{-3}$ an extrasolar planet is a gas balloon (not discussed), theoretical limits on the density of the confirmed exoplanets 82 Eridani b would be: $1.4 \mathrm{R}_{\text {Earth }}<\mathrm{R}_{\mathrm{exo}}<2.2 \mathrm{R}_{\text {Earth }}$.

\section{Universal Geo-cosmic X-structure Level}

Table 1. Similarities at the Geo-cosmic X-structure of the Solar system natural satellites [1, 8, 10-11] in the legend: Satellite diameter [km] density [G. $\left.\mathrm{cm}^{-3}\right]$ albedo.

\begin{tabular}{|c|c|c|c|c|c|c|c|c|c|}
\hline $\begin{array}{l}\text { Reduced } \\
\text { semi-major }\end{array}$ & Jupiter & Saturn & Uranus & Neptune & Pluto & Mars & \multicolumn{3}{|c|}{ Number of similarities } \\
\hline \multirow[t]{2}{*}{4.5} & \multirow{3}{*}{$\begin{array}{l}\text { Rings: } \\
\text { Density } 1 \mathrm{G}^{\cdot} \mathrm{cm}^{-3} \text {, } \\
\text { albedo } 0.05\end{array}$} & \multirow[t]{3}{*}{ Pan 280.40 .115} & Cordelia 211.30 .08 & & & \multirow[b]{4}{*}{ Phobos 111.9} & $\begin{array}{l}\text { By } \\
\text { density } \\
8\end{array}$ & $\begin{array}{l}\text { By } \\
\text { diameter } \\
7\end{array}$ & $\begin{array}{l}\text { By } \\
\text { albedo } \\
7\end{array}$ \\
\hline & & & Ophelia 231.30 .08 & & & & \multirow{5}{*}{9} & \multirow{5}{*}{8} & \multirow{5}{*}{36} \\
\hline \multirow{4}{*}{9} & & & $\begin{array}{lll}\text { Bianca } 24 & 1.30 .08 \\
\text { Kressida } 41 & 1.3 & 0.08 \\
\text { Desdemona } & 34 & 1.30 .08 \\
\text { Juliet } 53 & 1.3 & 0.08\end{array}$ & & & & & & \\
\hline & 300.860 .05 & Janus 180.0610 .81 & Portia 701.30 .68 & $\begin{array}{l}\text { Naiad } 60-100 \\
1.30 .57\end{array}$ & & & & & \\
\hline & 100.860 .05 & Mimas 4001.140 .6 & Rosalind 721.30 .08 & $\begin{array}{l}\text { Thalassa } 100 \\
1.3\end{array}$ & & \multirow[t]{2}{*}{0.01} & & & \\
\hline & Rings: & Atlas 320.430 .4 & Cupid 361.30 .07 & $\begin{array}{l}\text { Деспина } 180 \\
1.30 .09\end{array}$ & & & & & \\
\hline
\end{tabular}




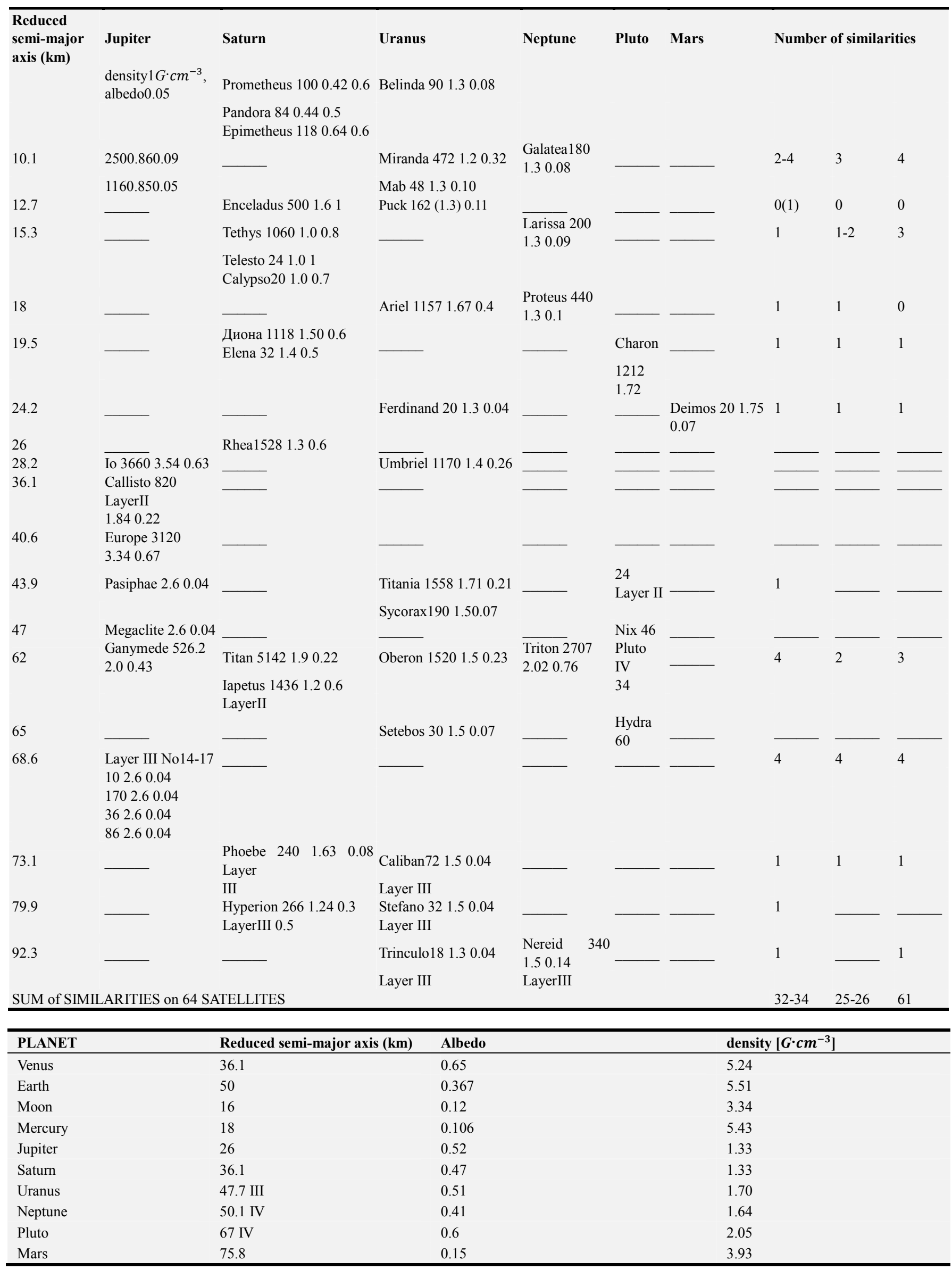




\section{Similarity at the Solar System Natural Satellites}

There are the following parameters similarities on planets: Mercury-Moon, Venus-Uranus, Jupiter-Rhea (Saturn) Mercury-Proteus (Neptune), Pluto-Triton-Titan, EarthGanymede (Jupiter) on Table 1.

\section{Discussion}

Mercury and the Moon on the Geo-cosmic universal Xstructure as related to structural levels of the reduced semimajor axis of the orbit $18 \mathrm{~km}$. So their property could theoretically be very close. The following is a list of the similarities.

- Mercury's diameter-4878 $\mathrm{km}$ at $3476 \mathrm{~km}$ from the Moon;

- «...It was thought that Mercury rotates synchronously and it (approximately-A.G.) always faces to the Sun by one hemisphere, just as the Moon made by one side to the Earth» [12];

- Mercury's albedo 0.106 to 0.12 at the Moon;

- "The measurement showed that spectrophotometric properties of surface rocks of Mercury's many areas of mainland rocks resemble the Moon, though somewhat lighter than their"([12],p.7);

- "Chapter 2. LUNAR LANDSCAPES of Mercury" [12];

- Mercury and the Moon, related to meteorite age [14], close to the geological structure.

- Should be mentioned that a list of similarities only applies to border on the surface. While theoretically the power of the crust- $40 \mathrm{~km}$ of the Moon and $46 \mathrm{~km}$ from the Jupiter's sea in the area of Mercury [10] the differences are substantial (see also [12]). Recent measurements of the Moon's crust power give $37 \mathrm{~km}$ (data web site: Wikipedia) that is within the permissible variation in theory [10-11].

With the help of computer calculations was simulated the process of planetary formation of clouds of Protoplanetary bodies, and it was shown that patterns of structure of the solar system, such as the separation of the planets on the two big groups, consistently observed in the majority of cases ([12], p.37).

The New Horizons team notes that Triton, likely a former Kuiper Belt planet captured by Neptune, was considered the best analog for Pluto prior to the July 14 flyby [16]. The team now believes that the geologies of Triton and Pluto are more different than similar, but will know more as additional data are returned.

Commentary: The new X-structure method (see Appendix B) for the case of our satellite Moon give $40 \mathrm{~km}$ it has recently been confirmed by measurement data, according to which the thickness of the crust of the Moon is equal to an average of $37 \mathrm{~km}$. Whereas the first measurements with the help of Seismometer in-ground landing spacecrafts gave only $30 \mathrm{~km}$. Therefore, these new data coincide within the $2 \sigma-$ uncertainty with theory.
X-structure of Titan (radius $1304 \mathrm{~km}$ ): Ice crust of Titan's Ruakh depression $30 \mathrm{~km}$ (Table B7). Ice crust of Titan's Equateur province with a large Graben $27 \mathrm{~km}$ (Table B8). Whereas the ice crust Pluto (radius $1187 \mathrm{~km}$ ) thickness at pole is $18.2 \mathrm{~km}$ almost twice thinner and only taking into account the thickness of the crust to Layer II X-structure on the lower elevation give $35.2 \mathrm{~km}$. Of course, that the geologic structure of Pluto and Triton must have great differences.

\section{Conclusions}

(1) On the Figures 1-4 exoplanet's points are exactly on graph of the Geo-cosmic $\mathrm{X}$-structure within the permissible deviation of the length despite of a fairly high density. As can be seen in all three cases, parametric exoplanets points on scheme well consistent with the universal Geo-cosmic structure $[1,11]$ for various radii of the central star.

(2) Important when comparing theory with observed three exoplanets orbit parameters 82 Eridani is the largest major axes all three exoplanets are exactly at the boundary set theory. The density closest to the 82 Eridani extrasolar planet is additional fixed by the largest plateau of the first peak Figure 2 due to the small off set to the left. However, the theory is valid and the interval of less than $3 \mathrm{G}^{\cdot} \mathrm{cm}^{-3}$, while the more distant orbits, one of which coincided with the orbit of Mercury, the theoretically permission interval of density is small and coincided with the experimental dispersion. Because the values of the density less than $1 G^{\circ} \mathrm{cm}^{-3}$ an extrasolar planet is a gas balloon (not discussed), theoretical limits on the density of the confirmed exoplanets 82 Eridani b would be: $1.4 \mathrm{R}_{\text {Earth }}<\mathrm{R}_{\text {exo }}<2.2 \mathrm{R}_{\text {Earth. }}$.

(3) The 82 Eridani star system weight 0.70 the Sun's mass and radius of 0.92 the Sun's radius distant 19.7 light years from Earth discovered and supposed three exoplanets $82 \mathrm{G}$. Eridani b, c, d. From the measured albedo 0.3 , the surface temperature of exoplanets $115^{\circ} \mathrm{C}$ is allowed this stellar system top grade of habitability. The major semi axis of orbits in a way binding on the radius of the central star (scaling factor $\mathrm{K}=0.92$ order 1 ) identified as $6.58 \pm 0.1,11.1 \pm 0.16,19.0 \pm 0.32 \mathrm{~km}$ for extrasolar planets $b, c, d$ respectively.

(4) The range of density values of extrasolar planets b, c, d respectively their masses (2.52 \pm 0.3$) \mathrm{M}_{\text {Earth }},(2.52 \pm 0.3) \mathrm{M}_{\text {Earth }}$ and (4.7 \pm 0.6$) \mathrm{M}_{\text {Earth }}$ are exactly corresponded to the weight jumps diapasons of the X-structure. In this field within the spread the Universal Geo-cosmic X-structure of the planets in the Solar system gives the following values for the radii of extrasolar planets b, c, d 82 Eridani - $10880 \mathrm{~km}, 9860 \mathrm{~km}$ and $10720 \mathrm{~km}$ respectively.

(5) There are far not all exoplanets parameters such as density and radius defines. In all these indeterminate cases it may be possible productive use of the Geo-cosmic universal $\mathrm{X}$-structure of the Solar system $[1,11]$.

(6) The highest sum of similarities is the sum on albedo, almost equal to the number of satellites. This means that the structural levels of the Geo-cosmic X-structure of the Solar 
system are grouped directly observable way. During of the parade planets, for example.

(7) Sum of similarities on density reaches over $50 \%$ of the number of natural satellites.

(8) The sum of the similarities on diameters is not much less than the sum of similarities in density. There are similarities and geological structures of the satellites (see e.g. [14]).

(9) Comparative characteristics between the Titius-Bode systems Lunar law modification on 24 moons and method of the Geo-cosmic Universal X-structure of Solar system planets and satellites on all known 178 natural satellites: it turned out that the accuracy of orbital parameters of TitiusBode law modification error at 5-7 times rougher, and only in one case, four satellites of Uranus is within the $2 \sigma$ with respect to $\sigma$ of $\mathrm{X}$-structure.

(10) On pictures of the surface of Pluto and Charon the crust thickness was determined. Crust of Pluto from pole $21 \pm 1.7 \mathrm{~km}$ The ice crust Charon thickness at pole is $18.2 \pm 1.5 \mathrm{~km}$.

(11) The nearest planet found recently in the system of red dwarf Proxima Centauri b with a radius of $1 / 7$ of the Sun's radius [13]. Orbital radius exoplanets is equal to $1 / 20 \mathrm{AU}$ is 5 times the diameter of the Sun. X-structure of this exoplanet Proxima Centauri b has great similarities with Mercury, even at $5 \mathrm{G} / \mathrm{cm}^{3}$ density. Smaller values of density are also allowed to spread. As you can see this is consistent with the assumption of Bruges and his colleagues [13] on the density exoplanets Proxima Centauri b, as well as the similarities with Mercury.

\section{Appendix A: Lunar Systems Titius-Bode Low Modification}

Table A1. Uranus ${ }^{2}$.

\begin{tabular}{llllll}
\hline Satellite & & No & $\mathbf{A}^{\text {a }}($ day) & $\mathbf{B}^{\mathbf{b}}($ day) & $\mathbf{C}^{\mathbf{c}}($ thousandkm) \\
\hline Uranus V & Miranda & 1 & 1,0931 & 1,4135 & $-19,6$ \\
Uranus I & Ariel & 2 & 2,4485 & 2,5204 & $-3,6$ \\
Uranus II & Umbriel & 3 & 5,4848 & 4,1442 & 57,4 \\
Uranus IV & Oberon & 4 & 13,463 & 12,286 & 38.1 \\
Standard deviation & & & & $70.291 \pm 210$ thousand $\mathrm{km}$ \\
\hline
\end{tabular}

${ }^{a} A$ is Calculation result ${ }^{2}$ (orbital period, day).

${ }^{\mathrm{b}} B$ is Factually ${ }^{2}$, day.

${ }^{c} C$ is Re-calculation from periods (day) to orbit radius:

orbital radius error (thousand $\mathrm{km}$ ).

Table A2. Jupiter ${ }^{2}$.

\begin{tabular}{llllll}
\hline Satellite & & No & $\mathbf{A}^{\mathbf{a}}(\mathbf{d a y})$ & $\mathbf{B}^{\mathbf{b}}$ (day) & $\mathbf{C}^{\mathbf{c}}($ thousand $\mathbf{k m})$ \\
\hline Jupiter V & Amalthea & 1 & 0,9013 & 0,4982 & 145.210 \\
Jupiter I & Io & 2 & 1,8296 & 1,7691 & 9.600 \\
Jupiter II & Europa & 3 & 3,7142 & 3,5512 & 20.540 \\
Jupiter III & Ganymede & 4 & 7,5399 & 7,1546 & 38.415 \\
Jupiter IV & Callisto & 5 & 15,306 & 16,689 & -103.862 \\
Jupiter VI & Himalia & 9 & 259,92 & 249,72 & 311.844 \\
Standard deviation & & & & $70.291 \pm 210$ Thousand km \\
\hline
\end{tabular}

${ }^{a} A$ is Calculation result ${ }^{2}$ (orbital period, day).

${ }^{\mathrm{b}} B$ is Factually ${ }^{2}$, day.

${ }^{c} C$ is Re-calculation from periods (day) to orbit radius: orbital radius error (thousand $\mathrm{km}$ ).

${ }^{2}$ Web-site:https://fr.wikipedia.org/wiki/Loi_de_Dermott
Table A3. Saturn ${ }^{2}$

\begin{tabular}{llllll}
\hline Satellite & & \multicolumn{1}{c}{ No $\mathbf{A}^{\text {a }}$ (day) } & $\mathbf{B}^{\mathbf{b}}(\mathbf{d a y})$ & $\mathbf{C}^{\mathbf{c}}($ thousandkm) \\
\hline SaturnI & Mimas & 1 & 0,7345 & 0,9424 & $-27,4$ \\
SaturnII & Enceladus & 2 & 1,168 & 1,3702 & $-23,4$ \\
SaturnIII & Tethys & 3 & 1,8571 & 1,8878 & $-3,2$ \\
SaturnIV & Dione & 4 & 2,9528 & 2,7369 & 19,8 \\
SaturnV & Rhea & 5 & 4,6949 & 4,5175 & 13,8 \\
SaturnVI & Titan & 7 & 11,869 & 15,945 & $-208,4$ \\
& & 8 & 18,872 & & \\
SaturnVIII & Iapetus & 11 & 75,859 & 79,33 & $-106,8$ \\
Standarddeviation & & & & $70.291 \pm 210$ Thousandkm \\
\hline
\end{tabular}

${ }^{a} A$ is Calculation result ${ }^{2}$ (orbital period, day).

${ }^{\mathrm{b}} B$ is Factually ${ }^{2}$, day.

${ }^{c} C$ is Re-calculation from periods (day) to orbit radius:

orbital radius error (thousand $\mathrm{km}$ ).

Table A4. Lunar systems Titius-Bode law modification (Table A1.-A3) and the Geo-cosmic Universal X-structure of Solar system planets and satellits.

\begin{tabular}{lllllll}
\hline $\mathbf{A}^{\mathbf{a}}$ & $\mathbf{B}^{\mathbf{b}}$ & $\mathbf{C}^{\mathbf{c}}$ & $\mathbf{D}^{\mathbf{d}}$ & $\mathbf{E}^{\mathbf{e}}($ thousand $\mathbf{k m})$ & $\mathbf{F}^{\mathbf{f}}($ thousand $\mathbf{k m})$ & $\mathbf{G}^{\mathbf{g}}(\mathbf{\%})$ \\
\hline Jupiter-21 & 21 & 0 & 67 & 29 & $70 \pm 210$ & $7 \sigma_{\mathrm{X}}$ \\
Saturn-31 & 31 & 0 & 62 & 15 & $-48 \pm 75$ & $5 \sigma_{\mathrm{X}}$ \\
Uranus-26 & 26 & 1 & 27 & 15 & $18 \pm 36$ & $2.4 \sigma_{\mathrm{X}}$ \\
Neptune-14 & 14 & 0 & 14 & 10 & - & - \\
Pluton-5 & 5 & 0 & 5 & 1,5 & - & - \\
\hline
\end{tabular}

${ }^{a} A$ is Lunar systems.

${ }^{\mathrm{b}} B$ is The number of moons with the completed X-structure.

${ }^{c} C$ is The number of Diswrite.

${ }^{d} D$ is The number of moon total on $9 / 05 / 2015$.

${ }^{e} E$ is $\mathrm{X}$-structure standard deviation $\sigma_{\mathrm{X}}$ (thousand $\mathrm{km}$ ).

${ }^{f} F$ is Titius-Bode law modification standard deviation (thousand $\mathrm{km}$ ).

${ }^{g} G$ is Titius-Bode law modification error (\%).

\section{Appendix B: Thickness of the Crust of Pluto and Charon According to the Spacecraft New Horizons Data}



Figure B1. Pluto. Plot with mountain near the pole. Data (Web-sait: http://www.nasa.gov/feature/nasa-s-new-horizons-sees-more-detail-as-itdraws-closer-to-pluto/) 16.07.2015.

B. 1. Method for processing imagery of the surface. Preliminary information

It had nothing to do with interesting studies of Geologists (see [15]) drew attention to the significant impact of weak inhomogeneities gravitation field of the Earth on the formation of geological structures and deposits in the geological time scale. On the other hand, of course, you can assume that something similar may well occur in condition $\mathrm{s}$ of non-equilibrium of rocks. So the emergence of concepts of synergetics of geological systems, it was only a matter of 
time. Ask nown the free particles drag geological body should accumulate over it and increase the density of the overlying rocks, forming a kind of a pillar. Free movement of such particles is under the influence of gravity on capillary channels, micro and macro cracks, usually filled with water. This osmosis is the process of gravity transfer of solute molecules on the mechanism of gravitational motility. Difference between the soliton mechanism of a transport substance and osmosis is that the transfer may take place in a solid. Excessive pillar density depends on the gravity anomaly forming object and a number of geological factors. Estimated calculation lends itself to the potential energy of an object, which can go on moving the substance in gravitation field. Transport of material inside the pillar leads to the Earth decomposition and the surrounding rocks, i.e. to pillar with negative excess density. Exploration practice enough: It is known to increase the gravity anomalies due to pillar not dense rock above the deposits of hydrocarbons; revealed the presence of pillars within creasing density up to $20-50 \mathrm{~kg}$ per tone relative to the enclosing rocks above the ore layers, etc. When, any newly formed object makes a destabilizing factor. All this illustrates the prospect application of synergetic to transport substances in biological systems to geological processes. Characteristic for this process of transferring nervous squid by soliton HodgkinHuxley gets its counterpart transport material by the gravity diffusion soliton of Universal Geo-space X-structure of crust and upper mantle [1]. Unlike [15] transport substances are not only water, but also in the crystal lattice, only at a lower rate. With higher speed can occur, as it is known, abnormal exit into the metal of helium-3. It is with this new mechanism for transport of a substance due to the extremely small amount of helium-3 in the surface layers: at the helium-3 production on the outer edge of the terrestrial kernel density gradient is formed under the influence of which is formed the gravity diffusion helium soliton. There is a threshold value for the local in homogeneities of gravitation field for each class of particles. When this effect proportionality gravity mobility to the mass of an atom or compound, most electrically move able protons and hydroxyl ions can be portable by the soliton slower than more massive elements. It turns out that in the Earth's crust and mantle is located close to the surface and accumulate heavy chemical elements and compounds-it is precisely this is obliged to rise to the surface feature to the geothermal gradient in the platforms. Whereas, the lighter elements are positioned closer to the sole of the crust. All this takes place in each of the segments is in the crust and upper mantle in two layers to $700 \mathrm{~km} \mathrm{[1].}$ According to [1] the motion of the soliton is happening in real time. It is through the soliton motion theoretically found [1], that the annual displacement soles crust for almost the whole of Russia occur as a result of thermal expansion of soles in contact with the underlying rocks mantle layer. Of course, the notion of gravity diffusive soliton applies the greatest planetary-scale fractures.

Planet Earth represents a single material system all parts which both internal and external develop interlinked and mutually determined. In recent decades geologists realized that the device of the surface of our planet there is nothing like geomorphological expression of its internal structure, and that there are direct links between tectonic elements and forms of relief of the Earth's crust [15]. In General, the problem is reduced to the cognition of morph-tecto-Genesisthe process of the Earth development and its tectonic terrain. It is recognized that the presence of these links allows you to decipher the tectonic structure of the Earth.

In terms of geological research method, communication problems and deep subsurface tectonic structures geologist have study only available horizons of the Earth's crust and the underlying it judged only according to the geophysics and geochemistry. In its interpretation of the information of other Earth Sciences geologist could rely on reliable geotectonic concept, but that is not yet created, and practice requires its creation [15]. The urgency of this task has been reflected in numerous publications as well as in the Organization of the International Association-the International Geophysical project

You can expect that a gap in our knowledge will be remedied. But as is clear from the content of the draft establish patterns of communication forms of relief of the Earth's surface with tectonic structures of the upper horizons of the Earth's lithosphere was not foreseen in it.

According to the Universal Geo-space X-structure of the Earth crust and upper mantle [1] crust thickness can be determined directly on the picture surface. This conclusion is based on the public views on the izostatics relationship between a relief of a surface and of the sole structure of the crust in the reflection [1]. Also Geophysicists known about proportionality between the transverse size and the depth of the ore body. This method has been found a surprising similarity between X-structure of the terrain of the Canadian Shield III and Ukrainian Shield (on histograms of widths of gravity anomalies [1]) with the characteristic uncertainty $14 \%$, under the same conditions on the geographical latitude. It should be noted that, in practice, unlike the theoretical curve of Planetary fracture does not mean fallacy results. Of course if all operations was made correctly. Any deviations will be handled according to the theoretical provisions as a result of the physic-chemical complexity of the plot. In fact, these deviations are determined the chemical composition of the rocks composing, etc. features.

There are several other methods to search for the depth of the deposits and geological structures in the arsenal of the Universal Geo-space X-structure of the Earth crust and upper mantle in addition to the event-handling method for the pictures surface: method for the determination of geological cross-section, areas between neighboring contour bark thickness, etc. In the face of the Earth, these methods are used usually in conjunction that eliminates possible errors. However, in the context of other planets method for processing pictures surface, very innovating, not always has an alternative. The following Table B1 shows preliminary results using this method on a number of planets and moons to determine the thickness of the crust. 
Table B1. Summary of preliminary results of calculation the thickness of the crust of the Solar system planets and their moons ([10], Table8).

\begin{tabular}{|c|c|c|c|c|c|c|}
\hline$A^{a}$ & $\mathbf{B}^{\mathbf{b}}$ & $\mathrm{C}^{\mathrm{c}}(\mathrm{km})$ & $\bar{D}^{\mathbf{d}}$ & $E^{\mathrm{e}}(\%)$ & $\mathbf{F}^{\mathrm{f}}$ & $\mathrm{G}^{\mathrm{g}}(\mathrm{km})$ \\
\hline 1 & Earth & 12756 & & $0.9-1.6$ & 1 & 44.1 \\
\hline 2 & Moon & 3476 & $\begin{array}{l}\text { Lunar Alps, Rift } \\
\text { canyons }\end{array}$ & & 0.92 & 40 \\
\hline 3 & Mars & 6786 & -by lineaments, & $-0.4 \pm 1.6$ & 1.4 & 62 \\
\hline 4 & Mars & $6 / 86$ & -by profile; & $-0.1 \pm 1.5$ & 1.4 & 62 \\
\hline 5 & & & Mount Olympus & $0 \pm 1.6$ (up to $50 \mathrm{~km}$ ) & $1.3-1.4$ & 57 \\
\hline 6 & Venus & 12102 & The area of the crater Adams lithosphere & $-0.1 \pm 1.6$ & $1-1.14$ & 43 \\
\hline 7 & Mercury & 4878 & Sea of Jupiter & $0.5 \pm 4.2$ & 1.14 & 40 \\
\hline 8 & Jupiter/Europe & 3122 & & $0.2 \pm 5$ & 1 & 44 \\
\hline $\begin{array}{l}9 \\
10\end{array}$ & Uranus/Miranda & 480 & Canyons, the chevron relief long $132 \mathrm{~km}$ & $\begin{array}{l}0.6 \pm 3.4 \\
0.8 \pm 1.1\end{array}$ & 1.96 & 86.4 \\
\hline 11 & Sun & 1400000 & spot & 5 & 15 & $662^{*}$ \\
\hline
\end{tabular}

${ }^{a} A$ is No.

${ }^{\mathrm{b}} B$ is Planet/satellite.

${ }^{c} C$ is Diameter $(\mathrm{km})$.

${ }^{d} D$ is Plot surface.

${ }^{e} E$ is Deviation from a planetary fracture curve (PF) (\%).

${ }^{f} F$ is A scale factor $\mathrm{K}$.

${ }^{g} G$ is The thickness of the crust on the PF curve $(\mathrm{km})$.

*700 km depth of the bottom of the solar spots below the photosphere.

In Table B1 it can be seen that the thickness of the crust of the terrestrial planets, as well as Europe-satellite of Jupiter is about the same. While they all have a scale factor of order 1 . The most powerful bark Mars-62 km, which is associated within creased scale factor 1.4. The highest the thickness of the crust-satellite of Uranus Miranda $86 \mathrm{~km}$-also with a maximum scale factor of about 2 . This is comparable with most the thickness of the Earth crust up to $80 \mathrm{~km}$.

The theoretical value of the thickness of the crust from the Table B1 for the case of our satellite Moon $40 \mathrm{~km}$ it has the thickness of the crust of recently been confirmed by measurement data, according to which the thickness of the crust of the Moon is equal to an average of $37 \mathrm{~km}$. Whereas the first measurements with the help of Seismometer inground landing spacecrafts gave only $30 \mathrm{~km}$ [15]. Therefore, these new data coincide within the $2 \sigma$-uncertainty with theory.

Another example from the Table B1 - Miranda, a moon of Uranus. The estimated thickness of its crust, after processing the plot topography, Chevron was $82 \mathrm{~km}$. It's thicker than the absolute maximum of the Earth crust in the Himalayas. Miranda is an extraordinary interest to geologists because it is known even from the 80 's, in addition to the mysterious terrain of Chevron has $20 \mathrm{~km}$ of splitting. Recently the biggest hot of the splitting has been processed in crosssection, as is done on geological cross-section. The result is stunning: the thickness of the crust Miranda in the place of the splitting as appeared in 2 times less than on the chevron relief plot. It is the same as most planets and Earth. The impression that really, as expected and Geophysics, geology Miranda is glued together from multiple pieces. Let me remind you that it has been suggested that earlier Miranda was shattered to pieces, but then again gathered already on another orbit.

Finally, there is a third case, when we can look into the bowels of the cosmic bodies: this sun spots. Gravity on the surface of the solar photosphere horrifying in 27 times stronger than Earth's. 1 kg would have weighed on Sun $27 \mathrm{~kg}$.
In Table B1 shows the results of snapshot processing solar spots on the number of fibers and their length. The depth of the photosphere, the calculated thickness solar crust, amounted to $670 \mathrm{~km}$. It is not so much due to the strong gravitation. This value coincides with direct astronomical observations through the central sun spot hole.

Making all these required retreats you can go to the central topic of this section.

B. 2. Thickness of the crust of Pluto and Charon according to the spacecraft New Horizons data

Crust of Pluto from pole $21 \pm 1.7 \mathrm{~km}$. 16.07.2015. Reduced length is the length of the lineament, multiplied by 2.2 . Reduced thickness of crust on the X-structure- $46 \mathrm{~km}$. Reduced thickness of the crust to Layer II X-structure on the lower elevation is $35.2 \mathrm{~km}$.

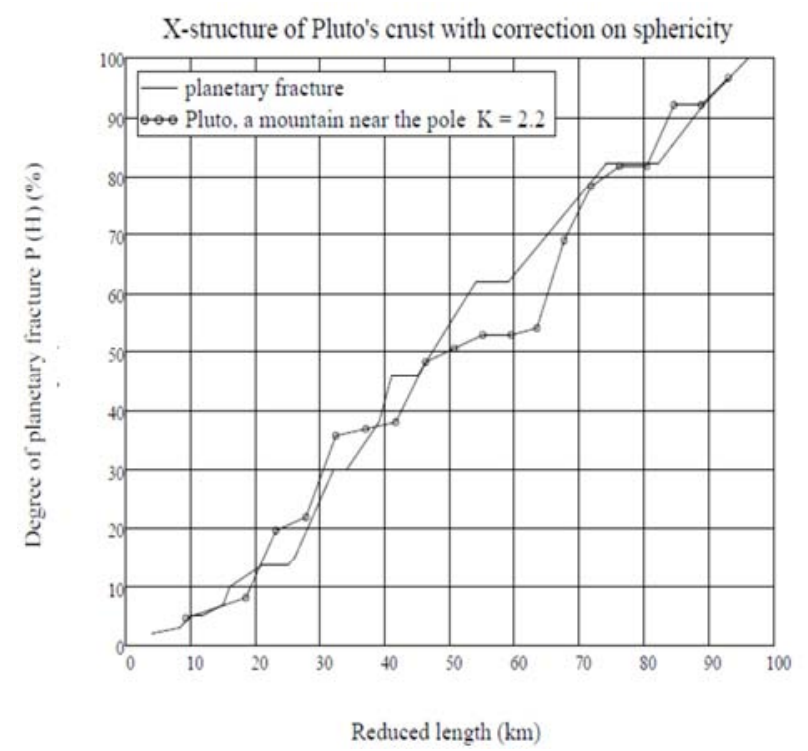

Figure B2. X-structure of Pluto on Figure B. 1. Reduced length is a Depth H $(\mathrm{km})$. 
The complete crustal thickness Pluto with ice $35.2 \pm 1.5 \mathrm{~km}$. This new image on Figure B4 of an area on Pluto's largest moon Charon has a captivating feature-a depression with a peak in the middle, shown here in the upper left corner of the inset.



Figure B3. X-structure of Pluto on Figure B.1 LayerII. Reduced length is a Depth $H(\mathrm{~km})$.

At center is a canyon estimated to be 4 to 6 miles ( 7 to 9 kilometers) deep. Mission scientists are surprised by the apparent lack of craters on Charon. South of the moon's equator, however, relatively few craters are visible, indicating are latively young surface that has been reshaped by geologic activity.

The image shows an area approximately 240 miles (390 kilometers) from top to bottom, including few visible craters. "The most intriguing feature is a large mountain sitting in a moat" commented New Horizons' Geology, Geophysics and Imaging team [17]- "This is a feature that has geologists stunned and stumped".

This image gives a preview of what the surface of this large moon will look like in future close-ups from New Horizons spacecraft. This image is heavily compressed; sharper versions are anticipated when the full-fidelity data are returned to Earth.

The rectangle superimposed on the global view of Charon shows the approximate location of this close-up view.

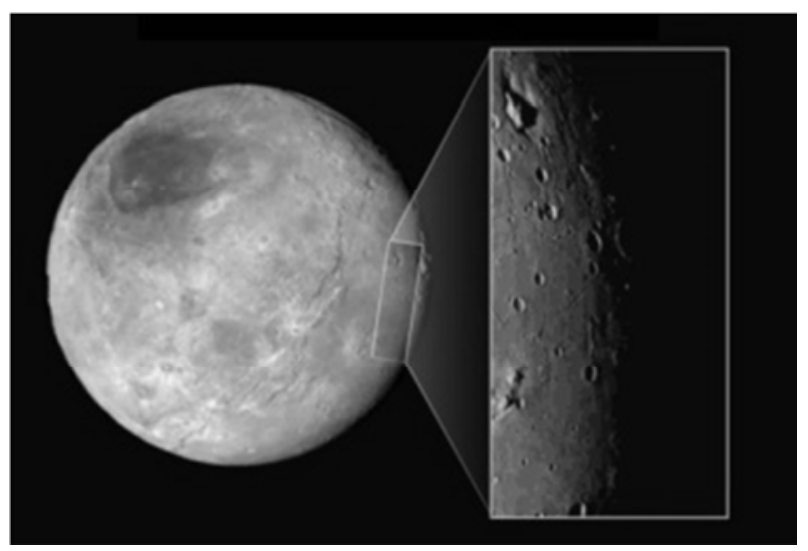

Figure B4. Charon. Plot with mountin the hollow about $300 \mathrm{~km}$ long. Data (Web-sait:http://www.nasa.gov/feature/nasa-s-new-horizons-sees-moredetail-as-it-draws-closer-to-pluto/)16.07.2015.
The image was taken on July 14, 2015, about 1.5 hours before closest approach to Pluto, from a range of 49,000 miles (79,000 kilometers). Directly on the image distance from top to bottom it was clarified to $372-375 \mathrm{~km}$ with uncertainty $\sigma=1.74 \mathrm{~km}$.

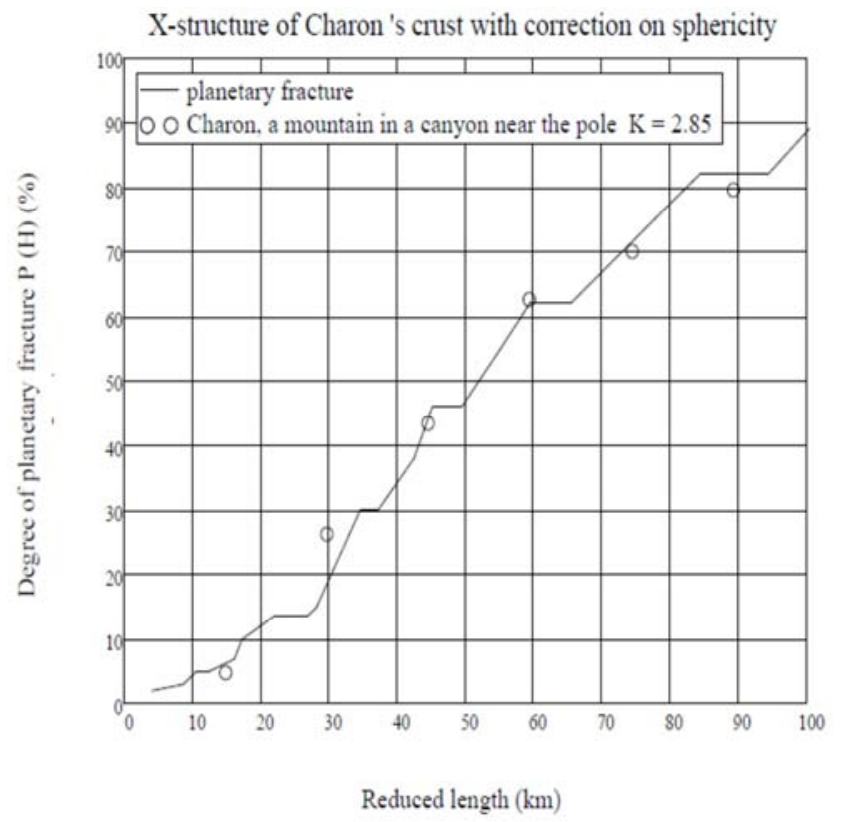

Figure B5. X-structure of Charon, a satellite of Pluto on Figure B.4. Reduced length is a Depth $H(\mathrm{~km})$.

\section{B3. LayerII}



Figure B6. X-structure of Charon on Figure A4. Layer II. Reduced length is a Depth $H(\mathrm{~km})$.

The ice crust Charon thickness at pole is $18.2 \pm 1.5 \mathrm{~km}$. 23.07.2015.

Reduced length is the length of the lineament, multiplied by 12.5. Reduced thickness of the crust to Layer II X- 
structure on the lower elevation is $35.2 \mathrm{~km}$.

The complete crustal thickness Charon with ice $35.2 \pm 1.5$ $\mathrm{km}$. The Moon is $37 \mathrm{~km}$.

The diameter of Pluto, according to the spacecraft New Horizons data [17] is $2370 \mathrm{~km}$ (uncertainty in 20 kilometers) was 18.5 percent of the Earth's diameter and Charon-1208 kilometers (9.5 percent) [17].

\section{B. 4. Discussion}

Scale factor $\mathrm{K}=12.5$ layer II Charon Figure B6 imagine how $3 \times 4$. Three is the transition rate to the first layer of crust according to the $\mathrm{X}$-structure. The temperature at the surface of Charon 53K [13]. It's in 5.3 times less $283 \mathrm{~K}$ on the surface of the Earth. The resulting higher multiplier of 4 just fits this scale on the X-structure formula [1] taking into account the small variations of the gravity field jump.

The first layer of Pluto on Figure B1-B.2 K = 2.2, but his temperature under snapshot [17] infrared light from the pole above-100K. Therefore, the absolute value of $\mathrm{K}=2.2$ taking into account variations in the gravity field jump Pluto is also acceptable.

Table B6. Pluto's $375 \mathrm{~km}$ long plot with the mountain at pole Figure B1. $K=2.2$. Data [17]

\begin{tabular}{lllllll}
\hline $\mathbf{A}^{\mathbf{a}}$ & $\mathbf{B}^{\mathbf{b}}$ & $\mathbf{C}^{\mathbf{c}}$ & $\mathbf{D}^{\mathbf{d}}$ & $\mathbf{E}^{\mathbf{e}}$ & $\mathbf{F}^{\mathbf{f}}$ & $\mathbf{G}^{\mathbf{g}}$ \\
\hline 1 & 4.2 & 2 & 9.24 & 4.6 & 4 & 2 \\
2 & 8.4 & 3.5 & 18.48 & 8.05 & 8.3 & 3 \\
3 & 10.5 & 8.5 & 23.1 & 19.55 & 10 & 5 \\
4 & 12.6 & 9.5 & 27.72 & 21.85 & 11.7 & 5 \\
5 & 14.7 & 15.5 & 32.34 & 35.65 & 15 & 6.9 \\
6 & 16.8 & 16 & 36.96 & 36.8 & 16 & 9.9 \\
7 & 18.9 & 16.5 & 41.58 & 37.95 & 21 & 13.6 \\
8 & 21 & 21 & 46.2 & 48.3 & 25 & 13.6 \\
9 & 23 & 22 & 50.6 & 50.6 & 26 & 14.9 \\
10 & 25 & 23 & 55 & 52.9 & 32 & 29.9 \\
11 & 27 & 23 & 59.4 & 52.9 & 34 & 29.9 \\
12 & 28.8 & 23.5 & 63.36 & 54.05 & 39 & 38 \\
13 & 30.7 & 30 & 67.54 & 69 & 41 & 46 \\
14 & 32.6 & 34 & 71.72 & 78.2 & 45 & 46 \\
15 & 34.6 & 35.5 & 76.12 & 81.65 & 50 & 55 \\
16 & 36.5 & 35.5 & 80.3 & 81.65 & 54 & 62 \\
17 & 38.4 & 40 & 84.48 & 92 & 59 & 62 \\
18 & 40.3 & 40 & 88.66 & 92 & 74 & 82 \\
19 & 42.2 & 42 & 92.84 & 96.6 & 82 & 82 \\
& & & & & 89 & 92 \\
& & & & & 96 & 100 \\
\hline
\end{tabular}

The standard deviation of the data from within the theory $\sigma=1 \%$

${ }^{a} A$ is No.

${ }^{\mathrm{b}} B$ is Lineament length $\mathrm{L}(\mathrm{km})$.

${ }^{c} C$ is The quoted number of lineaments to $L$ length of $\mathrm{P}_{\mathrm{A}}(\mathrm{H})(\%)$

${ }^{d} D$ is Reduced depth $\mathrm{H} 1=\mathrm{L} \cdot \mathrm{K}(\mathrm{km})$.

${ }^{e} E$ is The quoted number of lineaments to $\mathrm{H} 1$ length $2.3 \cdot \mathrm{P}_{\mathrm{A}}(\mathrm{H})(\%)$.

${ }^{f} F$ is Depth $\mathrm{H}(\mathrm{km})$.

${ }^{g} G$ is Degree of Planetary Fracture $\mathrm{P}_{\mathrm{X}}(\mathrm{H})(\%)$

\section{B5. X-structure of Triton in an analogy with Pluto}

The New Horizons team notes that Triton, likely a former Kuiper Belt planet captured by Neptune, was considered the best analog for Pluto prior to the July 14 flyby [16]. The team now believes that the geologies of Triton and Pluto are more different than similar, but will know more as additional data are returned.
Commentary: The new X-structure method for the case of our satellite Moon give $40 \mathrm{~km}$ it has recently been confirmed by measurement data, according to which the thickness of the crust of the Moon is equal to an average of $37 \mathrm{~km}$. Whereas the first measurements with the help of Seismometer inground landing spacecrafts gave only $30 \mathrm{~km}$. Therefore, these new data coincide within the $2 \sigma$-uncertainty with theory.

$\mathrm{X}$-structure of Titan (radius $1304 \mathrm{~km}$ ): Ice crust of Titan's Ruakh depression $30 \mathrm{~km}$ (Table B7). Ice crust of Titan's Equateur province with a large Graben $27 \mathrm{~km}$ (Table B8). Whereas the ice crust Pluto (radius $1187 \mathrm{~km}$ ) thickness at pole is $18.2 \mathrm{~km}$ almost twice thinner and only taking into account the thickness of the crust to Layer II X-structure on the lower elevation give $35.2 \mathrm{~km}$. Of course, that the geologic structure of Pluto and Triton must have great differences.

Table B7. Triton's $400 \mathrm{~km}$ long plot Ruakh depression ([14], Figure 36a).K=1.54. Data [17].

\begin{tabular}{|c|c|c|c|c|c|c|}
\hline$A^{a}$ & $\mathbf{B}^{\mathbf{b}}$ & $\mathbf{C}^{\mathbf{c}}$ & $D^{d}$ & $\mathbf{E}^{\mathbf{e}}$ & $F^{f}$ & $\mathbf{G}^{\mathbf{g}}$ \\
\hline 1 & 17.4 & 4 & 26.8 & 8.9 & 4 & 2 \\
\hline 2 & 21.9 & 7 & 33.7 & 15.5 & 8.3 & 3 \\
\hline 3 & 26.7 & 20 & 41.12 & 44.3 & 10 & 5 \\
\hline 4 & 35.6 & 28 & 54.82 & 62.07 & 11.7 & 5 \\
\hline 5 & 44.5 & 34 & 68.53 & 75.3 & 15 & 6.9 \\
\hline 6 & 80.1 & 37 & 123.4 & 82 & 16 & 9.9 \\
\hline 7 & & & & & 21 & 13.6 \\
\hline 8 & & & & & 25 & 13.6 \\
\hline 9 & & & & & 26 & 14.9 \\
\hline 10 & & & & & 32 & 29.9 \\
\hline 11 & & & & & 34 & 29.9 \\
\hline 12 & & & & & 39 & 38 \\
\hline 13 & & & & & 41 & 46 \\
\hline 14 & & & & & 45 & 46 \\
\hline 15 & & & & & 50 & 55 \\
\hline 16 & & & & & 54 & 62 \\
\hline 17 & & & & & 59 & 62 \\
\hline 18 & & & & & 74 & 82 \\
\hline \multirow[t]{3}{*}{19} & & & & & 82 & 82 \\
\hline & & & & & 89 & 92 \\
\hline & & & & & 96 & 100 \\
\hline
\end{tabular}

The standard deviation of the data from within the theory $\sigma=1 \%$

${ }^{a} A$ is No.

${ }^{\mathrm{b}} B$ is Lineament length $\mathrm{L}(\mathrm{km})$.

${ }^{c} C$ is The quoted number of lineaments to $L$ length.

${ }^{d} D$ is Reduced depth $\mathrm{H} 1=\mathrm{L} \cdot \mathrm{K}(\mathrm{km})$.

${ }^{e} E$ is The quoted number of lineaments to $\mathrm{H} 1$ length of $\mathrm{P}_{\mathrm{A}}(\mathrm{H})(\%)$.

${ }^{f} F$ is Depth $\mathrm{H}(\mathrm{km})$.

$G^{g}$ is Degree of Planetary Fracture $\mathrm{P}_{\mathrm{X}}(\mathrm{H})(\%)$.

Crust of Triton's Ruakh depression $30 \pm 1.5 \mathrm{~km} .16 .10 .2015$. Reduced length is the length of the lineament, multiplied by 1.54. Reduced thickness of crust on the X-structure- $46 \pm 1.5$ $\mathrm{km}$. Crust of Triton's Equateur province with a large Graben $27 \mathrm{~km}$. Whereas the ice crust Charon thickness at pole is 18.2 $\mathrm{km}$ and the thickness of the crust to Layer II X-structure on the lower elevation is $35.2 \pm 1.5 \mathrm{~km}$. 
Table B8. Triton's $400 \mathrm{~km}$ long plot Equateur province with a large Graben ([14], Figure 37). K=1.7. Data [17].

\begin{tabular}{lllllll}
\hline $\mathbf{A}^{\mathbf{a}}$ & $\mathbf{B}^{\mathbf{b}}$ & $\mathbf{C}^{\mathbf{c}}$ & $\mathbf{D}^{\mathbf{d}}$ & $\mathbf{E}^{\mathbf{e}}$ & $\mathbf{F}^{\mathbf{f}}$ & $\mathbf{G}^{\mathbf{g}}$ \\
\hline 1 & 9 & 3 & 15.3 & 4.5 & 4 & 2 \\
2 & 13.5 & 10 & 22.95 & 14.9 & 8.3 & 3 \\
3 & 18 & 18 & 30.6 & 26.9 & 10 & 5 \\
4 & 22.5 & 23 & 38.25 & 34.3 & 11.7 & 5 \\
5 & 27 & 32 & 45.9 & 47.8 & 15 & 6.9 \\
6 & 31.5 & 40 & 53.55 & 59.7 & 16 & 9.9 \\
7 & 36 & 40 & 61.3 & 59.7 & 21 & 13.6 \\
8 & 40.5 & 54 & 68.85 & 80.6 & 25 & 13.6 \\
9 & 44.9 & 55 & 76.33 & 82 & 26 & 14.9 \\
10 & 49.4 & 55 & 83.98 & 82 & 32 & 29.9 \\
11 & 54 & 61 & 91.8 & 91 & 34 & 29.9 \\
12 & 58.5 & 61 & 99.4 & 91 & 39 & 38 \\
13 & 63 & 61 & 107.1 & 91 & 41 & 46 \\
14 & 67.5 & 67 & 114.75 & 100 & 45 & 46 \\
15 & 72 & 67 & 122.4 & 100 & 50 & 55 \\
16 & & & & & 54 & 62 \\
17 & & & & & 59 & 62 \\
18 & & & & & 74 & 82 \\
19 & & & & & 82 & 82 \\
& & & & & 96 & 92 \\
& & & & & & 100 \\
\hline
\end{tabular}

The standard deviation of the data from within the theory $\sigma=1 \%$

${ }^{a} A$ is No.

${ }^{\mathrm{b}} B$ is Lineament length $\mathrm{L}(\mathrm{km})$.

${ }^{c} C$ is The quoted number of lineaments to $L$ length.

${ }^{d} D$ is Reduced depth $\mathrm{H} 1=\mathrm{L} \cdot \mathrm{K}(\mathrm{km})$.

${ }^{e} E$ is The quoted number of lineaments to $\mathrm{H} 1$ length of $\mathrm{P}_{\mathrm{A}}(\mathrm{H})(\%)$.

${ }^{f} F$ is Depth $\mathrm{H}(\mathrm{km})$.

$G^{g}$ is Degree of Planetary Fracture $\mathrm{P}_{\mathrm{X}}(\mathrm{H})(\%)$.

Crust of Triton's Equateur province with a large Graben $27 \pm 1.5 \mathrm{~km}$. 16.10.2015. Reduced length is the length of the lineament, multiplied by 1.7 . Reduced thickness of crust on the X-structure- $46 \mathrm{~km}$.

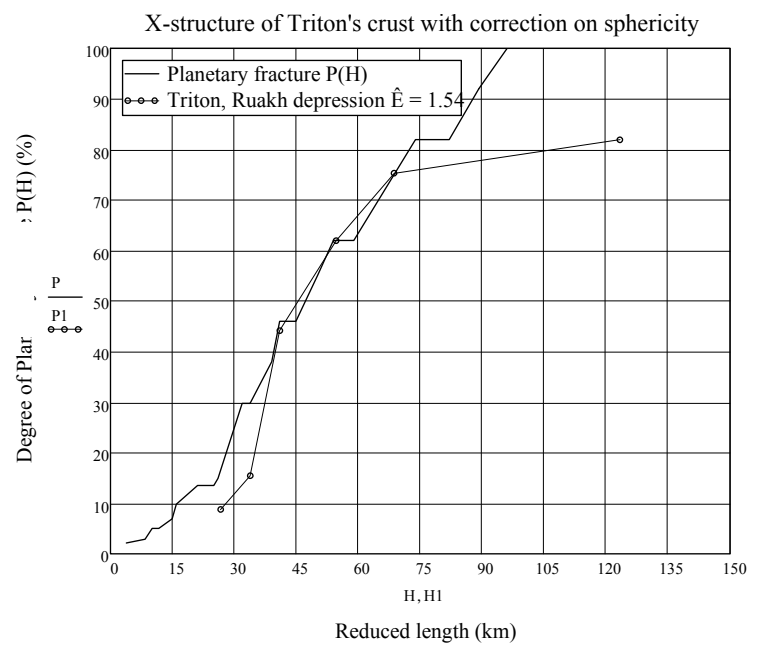

Figure B7. X-structure of Triton, Ruakh depression on ([14], Figure 36a). $K=1.54$. Reduced length is a Depth $H(\mathrm{~km})$.

The new X-structure method for the case of our satellite Moon give $40 \mathrm{~km}$ it has recently been confirmed by measurement data, according to which the thickness of the crust of the Moon is equal to an average of $37 \mathrm{~km}$. Whereas the first measurements with the help of Seismometer in- ground landing spacecrafts gave only $30 \mathrm{~km}$. Therefore, these new data coincide within the $2 \sigma$-uncertainty with theory.

X-structure of Triton: Ice crust of Triton's Ruakh depression $30 \pm 1.5 \mathrm{~km}$. Ice crust of Triton's Equateur province with a large Graben $27 \pm 1.5 \mathrm{~km}$. Whereas the ice crust Pluto thickness at pole is $18.2 \pm 1.5 \mathrm{~km}$ almost twice thinner and only taking into account the thickness of the crust to Layer II X-structure on the lower elevation is $35.2 \pm 1.5 \mathrm{~km}$.



Figure B8. X-structure of Triton, Equateur province with a large Graben on ([14], Figure 37).K=1.7. Reduced length is a Depth $H(\mathrm{~km})$.

\section{References}

[1] SYROMYATNIKOV A. G., \& ZAKOLDAEV Yu. A., Depth rock distribution according to the Geospace universal Xstructure of Earth crust and high mantle (Petropolis, SaintPetersburg, 2011).

[2] NERUCHEV S. G., Uranium and life in the history of the Earth (VNIGRI, Saint-Petersburg, Russia, 2007).

[3] CASSAN A., et al., Nature, 481 (7380), (2012) 167.

[4] DOYLE L. R., CARTER J. A., FABRYCKY D. C., et al., Science, 333 (6049), (2011) 1602.

[5] Special Issue: Exoplanets (3 May 2013), Science; SEAGER S., Science 340 (6132), (2013) 577; BHATTACHARJEE Yu., Science, 340 (6132), (3 May 2013) 542; BORUCKI William J., AGOL Eric, FRESSIN Francois, KALTENEGGER Lisa et al., Kepler-62: A Five-Planet System with Planets of 1.4 and 1.6 Earth Radii in the Habitable Zone, Science, 340 (6132), (3 May 2013) 587; HOWARD Andrew W., Science, 340 (6132), (3 May 2013) 572.

[6] SEAGER Sara, Science, 340(6132), (3 May 2013) 577.

[7] Web-site: https://en.wikipedia.org/wiki/Exoplanet.

[8] SYROMYATNIKOV A. G., Physical effects of conformal gauge theory of gravitation (LAP Lambert Academic Publishing GmbH\&Co. KG, Saarbrucken, Germany, 2012).

[9] SYROMYATNIKOV A. G., Int. J. Geom. Meth. Mod. Phys., Vol. 12, No. 10 (2015) 1550080 (1-47 pages).

[10] SYROMYATNIKOV A. G., The X-structure of solar system planets, (2011). DOI: 10.17686/sced_rusnauka_2011-218. 
[11] SYROMYATNIKOV A. G., Geockosmic universal Xstructure of the planets and satellites of the solar system. Report-forecast: Pluto-2015, Russian-Chinese scientific journal "sodruzhestvo" No. 3(3), /Physics and mathematics (2016) 115-124.

[12] KSANFOMALITY L. V., Parade of planets (Nauka. Fizmatlitgiz, Moskow, 1997).

[13] BRUGGER Bastien, MOUSIS Olivier, DELEUIL Magali \& LUNINE Jonathan I., Possible internal structures and composition of Proxima Centauri b, Accepted for publication in the Astrophysical Journal Letters, Vol. 12/16/11 (2016); arXiv: 1609.09757.
[14] KATTERFELD Gen. N., Five lectures on astro-geology and planetology (International Association of Planetologists, S.-Pb, 2002).

[15] KRIVOLUTSKII A. E., Reljef I nedra Zemli [Relief and The Bowels Of The Earth] (Izd-vo "Misl", Moscow, 1977).

[16] STERnS. A., BAGENAL F., ENNIKO K. et al, The Pluto system: Initial results from its exploration by New Horizons, Science, Vol. 350 No. 6258 (16 October 2015). DOI:10.1126/science.aad1815.

[17] Web-sait: http://www.nasa.gov/feature/nasa-s-new-horizonssees-more-detail-as-it-draws-closer-to-pluto/. 